\title{
The role of heat effects in the process of formation of color centers in LiF during filamentation of femtosecond laser pulses
}

\author{
A.V. Kuznetsov ${ }^{1}$, V.P. Dresvyansky ${ }^{1}$, O. Bukhtsoozh ${ }^{2}$, S. Enkhbat ${ }^{2}$, and E.F. Martynovich ${ }^{1}$ \\ ${ }^{1}$ Irkutsk branch of the Institute of Laser Physics SB RAS, 664033 Irkutsk, Russia \\ ${ }^{2}$ Institute of Physics and Technology, Mongolian Academy of Science, 210651 Ulaanbaatar, \\ Mongolia
}

\begin{abstract}
The possibility of the influence of thermal effects on the formation of color centers during laser femtosecond filamentation in a $\mathrm{LiF}$ crystal has been investigated theoretically. The conditions are formulated under which the influence of thermal effects can be noticeable.
\end{abstract}

\section{Introduction}

It is known, that femtosecond laser filamentation in LiF crystal leads to generation of permanent point defects of crystal lattice including fluorescent $\mathrm{F}_{2}$ and $\mathrm{F}_{3}{ }^{+}$color centers $[1,2]$. These color centers form a stable three-dimensional snapshot of filaments. Owing to this fact, $\mathrm{LiF}$ is attractive for studying of filamentation in general. Besides of that, the processes of laser-induced defects formation in LiF are of particular interest.

A goal of this work is theoretical estimation of effect of material heating during filamentation of repeated laser pulses in LiF on both filamentation process and defects formation. Results of this theoretical study can determine directions for further experiments.

Heating of material can have a number of consequences:

- thermo-optic modification of refractive index (thermo-optic coefficient $\mathrm{dn} / \mathrm{dT}$ of $\mathrm{LiF}$ is about $\left.1.710^{-5} \mathrm{~K}^{-1}[3]\right)$,

- variation of efficiency of color centers creation,

- transformation of color centers,

- macroscopic destruction of the crystal lattice.

\section{Mathematical model}

We introduce a number of simplifying assumptions based on known experimental facts:

- energy transfer from electron subsystem to nuclear subsystem takes much shorter time than period of repetition of laser pulses, thus this time is neglected;

- the length of filaments is usually 2 orders higher, than their width, thus infinitively long filaments are considered;

- lateral profile of filaments is assumed to have Gaussian form. 
Under these assumptions an evolution of temperature distribution $T(x, y, z, t)$ after the action of a number of laser pulses can be described with two-dimensional heat diffusion equation with Gaussian initial condition

$$
T_{0}(x, y)=T_{\max } \exp \left(-\frac{x^{2}+y^{2}}{r_{0}^{2}}\right)
$$

The amplitude of Gaussian $T_{\max }$ is not known from experiment. From theoretical considerations we expect $T_{\max }$ to be below $50 \mathrm{~K}$ in fresh material (without absorbing defects). Additional absobtion of induced defects can result in higer initial tepperature.

\section{Results of simulations}

Simulation shows, that for initial width of profile about $2 \mu \mathrm{m}$ (typical width of color centers trace of single filament) the time of heat diffusion has an order of $10^{-6} \mathrm{~s}$. Thus, insignificant heat accumulation is expected for single filamentation of laser pulses with repetition rate below $10^{6} \mathrm{~Hz}$.

More substantial heat accumulation can take place in areas of multiple filamentation. A simulation was performed for square grid of $30 \times 30$ filaments with the grid step of $20 \mu \mathrm{m}$. Temperature rise about $40 \mathrm{~K}$ is obtained in the center of the grid after the action of $10^{4}$ laser pulses with $10^{3} \mathrm{~Hz}$ repetition rate.

\section{Conclusions}

Our theoretical results predict negligibly small accumulation of the heat during single filamentation in $\mathrm{LiF}$ with repetition rate of laser pulses below $10^{6} \mathrm{~Hz}$. However, short time $\left(10^{-6} \mathrm{~s}\right)$ substantial heating after each laser pulse is possible upon condition that induced defects additionally absorb light energy.

Substantial accumulation of the heat can be possible with lower repetition rate during multiple filamentation with high number of filaments.

The research is carried out within the projects SB RAS II.10.1.2 and RFBR 17-52-44015 Mong_a.

\section{References}

1. L.C. Courrol, R.E. Samad, L. Gomes, I.M. Ranieri, S.L. Baldochi, A.Z. de Freitas, N.D. Vieira, Opt. Express 12, 288-293 (2004).

2. E.F. Martynovich, A.V. Kuznetsov, A.V. Kirpichnikov, E.V. Pestryakov, S.N. Bagaev, Quantum Electron. 43, 463-466 (2013)

3. A. Feldman, D. Horowitz, R.M. Walker, and M.J. Dodge, "Optical Materials Characterization Final Technical Report," Nat. Bur. Standards Technical Note 993 (1979) 